\section{Rheumatoide Arthritis: Wie effektiv ist Treat-to- Target in der Praxis?}

Nekvindová L et al. Switching first-line targeted therapy after not reaching low disease activity within 6 months is superior to conservative approach: a propensity score-matched analysis from the ATTRA registry. Arthritis Res Ther 2021; 23: 11. doi: 10.1186/s13075-020-02393-8

Ziel der sogenannte Treat-to-Target-Strategie bei der rheumatoiden Arthritis (RA) ist die Remission oder zumindest die niedrige Krankheitsaktivität innerhalb von 6 Monaten nach Therapiebeginn. Wird dieses Ziel nicht erreicht, muss die medikamentöse Behandlung angepasst werden. Ein tschechisches Forscherteam untersuchte nun, ob das Treat-to-TargetKonzept in der klinischen Praxis gegenüber der Standardversorgung Vorteile hat.

Die Wissenschaftlerinnen und Wissenschaftler interessierte dabei besonders, ob bei Patientinnen und Patienten, welche nach 6 Monaten noch keine Remission bzw. niedrige Krankheitsaktivität erreicht haben, nach einem Umstellen der gezielten Erstlinientherapie im Sinne des Treat-toTarget-Konzepts die Chancen auf ein Erreichen der Therapieziele nach 12 Monaten steigen. Hierzu werteten sie Daten des tschechischen ATTRA-Registers, einer prospektiven nationalen Beobachtungsstudie, welche die Sicherheit und Effektivität biologischer und gezielter synthetischer DMARDs (disease-modifying antirheumatic drugs) evaluiert, aus. In die Analyse flossen die Daten von 1275 erwachsenen RAKranken ein, welche zwischen 2012 und 2017 erstmals auf biologische oder gezielte synthetische DMARDs eingestellt und über mindestens 12 Monate nachbeobachtet worden waren. Auf der Basis des Therapieergebnis nach 6 Monaten bildete das Forscherteam 4 Gruppen:

- C1 (früher Switch): Umstellen der Therapie innerhalb der ersten 5 Monate (vor der Beurteilung des Therapieansprechens mit 6 Monaten)

- C2 (Therapieansprechen): Keine Therapieänderung, niedrigere Krankheitsaktivität nach 6 Monaten

- C3 (Switch entsprechen Treat-toTarget-Konzept): Kein Erreichen des Therapieziels nach 6 Monaten, daher Umstellen der Medikation

- C4 (Kein Switch entsprechend Treat-to-Target-Konzept): Kein Erreichen des Therapieziels nach 6 Monaten, aber trotzdem kein Umstellen der Medikation

Eine niedrigere Krankheitsaktivität lag per Definition bei einem DAS28 (disease activity score in 28 Gelenken) $\leq 3,2$ vor. Die Wissenschaftlerinnen und Wissenschaftler berechneten die Wahrscheinlichkeit für das Erreichen der Remission bzw. der niedrigen Krankheitsaktivität nach 12 Monaten in den Gruppen C3 und C4, wobei sie je 75 Patientinnen und Patienten dieser beiden Kollektiv 
mittels Propensity-Score-Matching miteinander verglichen.

\section{Ergebnisse}

Die Gruppen C1, C2, C3 und C4 umfassten 62 (4,9\%), 598 (46,9\%), 124 (9,7\%) sowie 491 (38,5\%) Personen im medianen Alter zwischen 51 und 55 Jahren. Das beste Therapieergebnis nach 12 Monaten stellte das Forscherteam in der Gruppe C2 fest: Nahezu 79\% dieser Patientinnen und Patienten befanden sich zu diesem Zeitpunkt in Remission oder hatten eine niedrige Krankheitsaktivität. In den Gruppen C1, C3 und C4 waren es dagegen nur 48, 40 bzw. 32\% der Personen. Der Vergleich der mittels Propensity-Score-Matching gebildeten Kollektive aus Gruppe C3 und C4 ergab: Die nach dem Treat-to-Target-Konzept behandelten Patientinnen und Patienten (C3) hatten im Vergleich zu den nicht gemäß dieser Strategie behandelten Personen (C4) eine um das 2,8-Fache höhere Wahrscheinlichkeit für ein Erreichen des Therapieziels (mindestens niedrige Krankheitsaktivität) nach $12 \mathrm{Mo-}$ naten $(p=0,005)$.

\section{FAZIT}

Im klinischen Alltag wird das Treat-to-Target-Konzept bei der Behandlung der RA nicht optimal umgesetzt, so das Fazit der Forscherinnen und Forscher. Ihre Studienergebnisse belegen die Überlegenheit dieser Strategie gegenüber der Standardversorgung: Die Umstellung auf ein anderes biologisches bzw. gezieltes synthetisches DMARD, sofern nach 6 Monaten nicht mindestens eine niedrige Krankheitsaktivität erreicht wird, erhöht die Chancen auf ein Erreichen der Therapieziele nach 12 Monaten deutlich.

Dr. med. Judith Lorenz, Künzell 